\title{
Covering minimal separators and potential maximal cliques in $\boldsymbol{P}_{\boldsymbol{t}}$-free graphs*
}

\author{
Andrzej Grzesik \\ Faculty of Mathematics and Computer Science, \\ Jagiellonian University, Kraków, Poland, \\ andrzej.grzesik@uj.edu .pl
}

\author{
Tereza Klimošová \\ Department of Applied Mathematics, \\ Charles University, Prague, Czech Republic, \\ tereza@kam.mff.cuni.cz
}

\author{
Marcin Pilipczuk \\ Institute of Informatics, \\ University of Warsaw, Poland, \\ marcin.pilipczuk@mimuw.edu.pl
}

\author{
Michał Pilipczuk \\ Institute of Informatics, \\ University of Warsaw, Poland, \\ michal.pilipczuk@mimuw.edu.pl
}

Submitted: Mar 27, 2020; Accepted: Jan 24, 2021; Published: Feb 12, 2021

(C) The authors. Released under the CC BY-ND license (International 4.0).

\footnotetext{
*The early stage of this research was done while Andrzej Grzesik held a post-doc position of Warsaw Centre of Mathematics and Computer Science (WCMCS), WCMCS supported a visit of Tereza Klimošová in Warsaw, Tereza Klimošová was supported by ANR project Stint under reference ANR-13-BS02-0007 and by the LABEX MILYON (ANR-10-LABX-0070) of Universite de Lyon, within the program "Investissements d'Avenir" (ANR-11-IDEX-0007) operated by the French National Research Agency (ANR). Ma. Pilipczuk was supported by the Polish National Science Centre grant UMO-2013/09/B/ST6/03136, and Mi. Pilipczuk was supported by the Foundation for Polish Science (FNP) via the START stipend programme.

The later stages of this research were supported as follows. Tereza Klimošová is supported by the grant no. 19-04113Y of the Czech Science Foundation (GAČR) and the Center for Foundations of Modern Computer Science (Charles Univ. project UNCE/SCI/004).

The research or other authors is a part of projects that have received funding from the European Research Council (ERC) under the European Union's Horizon 2020 research and innovation programme Grant Agreements no. 648509 (A. Grzesik), no. 714704 (Ma. Pilipczuk), and no. 677651 (Mi. Pilipczuk).

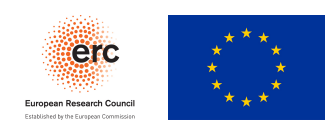




\begin{abstract}
A graph is called $P_{t}$-free if it does not contain a $t$-vertex path as an induced subgraph. While $P_{4}$-free graphs are exactly cographs, the structure of $P_{t}$-free graphs for $t \geqslant 5$ remains not well-undestood. On one hand, classic computational problems such as Maximum Weight Independent Set (MWIS) and 3-Coloring are not known to be NP-hard on $P_{t}$-free graphs for any fixed $t$. On the other hand, despite significant effort, polynomial-time algorithms for MWIS in $P_{6}$-free graphs [SODA 2019] and 3-Coloring in $P_{7}$-free graphs [Combinatorica 2018] have been found only recently. In both cases, the algorithms rely on deep structural insights into the considered graph classes.

One of the main tools in the algorithms for MWIS in $P_{5}$-free graphs [SODA 2014] and in $P_{6}$-free graphs [SODA 2019] is the so-called Separator Covering Lemma that asserts that every minimal separator in the graph can be covered by the union of neighborhoods of a constant number of vertices. In this note we show that such a statement generalizes to $P_{7}$-free graphs and is false in $P_{8}$-free graphs. We also discuss analogues of such a statement for covering potential maximal cliques with unions of neighborhoods.
\end{abstract}

Mathematics Subject Classifications: 05C69,05C75,05C85,68R10

\title{
1 Introduction
}

By $P_{t}$ we denote a path on $t$ vertices. A graph is $H$-free if it does not contain an induced subgraph isomorphic to $H$.

We are interested in classifying the complexity of fundamental computational problems, such as Maximum Weight Independent Set (MWIS) or $k$-Coloring for fixed or arbitrary $k$, on various hereditary graph classes, in particular on $H$-free graphs for small fixed graphs $H$. As noted by Alekseev [1], MWIS is NP-hard on $H$-free graphs unless every connected component of $H$ is a tree with at most three leaves. Similarly, 3-CoLORING is known to be NP-hard on $H$-free graphs unless every connected component of $H$ is a path [11]. On the other hand, it would be consistent with our knowledge if all the remaining cases actually led to polynomial-time solvability. This remains still unknown in spite of intensive work on the subject, which we review next.

That MWIS is polynomial-time solvable on $P_{4}$-free graphs (also known as cographs) follows from the observation that these graphs have bounded cliquewidth. A polynomialtime algorithm for MWIS on $P_{5}$-free graphs was proposed by Lokshtanov et al. [17], which was followed by a polynomial-time algorithm on $P_{6}$-free graphs due to the current authors [13]. Both these works heavily rely on the approach of potential maximal cliques, which of central interest in this work. It is also known that MWIS is polynomial-time solvable on claw-free graphs $[19,21]$ and on fork-free graphs [18], where the claw is $K_{1,3}$ and the fork is the claw with one edge subdivided once. For coloring problems, it is known that 3-Coloring can be solved in polynomial time on $P_{7}$-free graphs [3] and 4Coloring can be solved in polynomial time on $P_{6}$-free graphs [8]. It is also known that for every fixed $k, k$-COLORING is polynomial-time solvable on $P_{5}$-free graphs [15]. 
While the polynomial-time algorithms presented above are rather limited in generality, much more encouraging results are known if the requirement of polynomial running time is relaxed. In a very recent breakthrough, Gartland and Lokshtanov [10] have shown a quasipolynomial-time algorithm, with running time $n^{\mathcal{O}\left(\log ^{3} n\right)}$, for MWIS on $P_{t}$-free graphs, for every fixed $t$. The running time has been improved to $n^{\mathcal{O}\left(\log ^{2} n\right)}$ by Pilipczuk et al. [20], who also observed that the same technique can be used to give an $n^{\mathcal{O}\left(\log ^{2} n\right)}$-time algorithm for 3-ColORING on $P_{t}$-free graphs, for every fixed $t$. Let us note that these results were established after the announcement of this work, however they were inspired by techniques introduced in an earlier line of work on subexponential-time algorithms for problems in question $[2,6,12]$. It is still unknown whether MWIS can be solved in quasi-polynomial time on $H$-free graphs whenever every connected component of $H$ is a tree with at most 3 leaves, however Chudnovsky et al. [7] have given both a subexponential-time algorithm and a quasi-polynomial-time approximation scheme in this setting.

The state-of-art presented above shows a large gap between cases where quasi-polynomial-time algorithms are known, and cases where actual polynomial-time solvability has been established. It seems that there is a certain lack of a deeper understanding of the structure of $P_{t}$-free graphs for larger values of $t$, which prevents us from properly exploiting this structure in algorithm design. In this note we take a closer look at one property that appeared important in the algorithms for MWIS for $P_{5}$-free and $P_{6}$-free graphs $[17,16,13]$, namely the possibility to cover a minimal separator with a small number of vertex neighborhoods.

Let $G$ be a graph. For a set $S \subseteq V(G)$, a connected component $A$ of $G-S$ is a full component to $S$ if $N_{G}(A)=S$. A set $S$ is a minimal separator if it admits at least two full components. A set $F \subseteq\left(\begin{array}{c}V(G) \\ 2\end{array}\right) \backslash E(G)$ is a chordal completion if $G+F:=(V(G), E(G) \cup F)$ is chordal (i.e., does not contain an induced subgraph isomorphic to a cycle on at least four vertices). A set $\Omega \subseteq V(G)$ is a potential maximal clique (PMC) if there exists an (inclusion-wise) minimal chordal completion $F$ of $G$ such that $\Omega$ is a maximal clique of $G+F$. Potential maximal cliques and minimal separators are tightly connected: for example, a graph is chordal if and only if every minimal separator is a clique, and if $\Omega$ is a PMC in $G$, then for every connected component $D$ of $G-\Omega$ the set $N_{G}(D)$ is a minimal separator with $D$ being one of the full components.

A framework of Bouchitté and Todinca [4, 5], extended by Fomin, Todinca, and Villanger [9], allows solving multiple computational problems (including MWIS) on graph classes where graphs have only a polynomial number of PMCs. While $P_{5}$-free graphs do not have this property, the crucial insight of the work of Lokshtanov, Villanger and Vatshelle [17] allows modifying the framework to work for $P_{5}$-free graphs and, with more effort, for $P_{6}$-free graphs [13].

A simple, but crucial in [17], insight about the structure of $P_{5}$-free graphs is the following lemma.

Lemma 1 ([17]). Let $G$ be a $P_{5}$-free graph, let $S$ be a minimal separator in $G$, and let $A$ and $B$ be two full components of $S$. Then for every $a \in A$ and $b \in B$ it holds that $S \subseteq N_{G}(a) \cup N_{G}(b)$. 
The above statement is per se false in $P_{6}$-free graphs, but the following variant is true and turned out to be pivotal in [13]:

Lemma 2 (Lemma 4.5 of [13], Lemma 18 in the arXiv version). Let $G$ be a $P_{6}$-free graph, let $S$ be a minimal separator in $G$, and let $A$ and $B$ be two full components of $S$. Then there exist nonempty sets $A^{\prime} \subseteq A$ and $B^{\prime} \subseteq B$ such that $\left|A^{\prime}\right| \leqslant 3,\left|B^{\prime}\right| \leqslant 3$, and $S \subseteq N_{G}\left(A^{\prime}\right) \cup N_{G}\left(B^{\prime}\right)$.

That is, every minimal separator in a $P_{6}$-free graph has a dominating set of size at most 6 , contained in the union of two full components of this separator.

In Section 3 we extend the result to $P_{7}$-free graphs as follows.

Theorem 3. Let $G$ be a $P_{7}$-free graph and let $S$ be a minimal separator in $G$. Then there exists a set $S^{\prime} \subseteq V(G)$ of size at most 22 such that $S \subseteq N_{G}\left[S^{\prime}\right]$.

Theorem 3 directly generalizes a statement proved by Lokshtanov et al. [16, Theorem 1.3], which was an important ingredient of their quasi-polynomial-time algorithm for MWIS in $P_{6}$-free graphs.

Section 5 discusses a modified example from [16] that witnesses that no statement analogous to Theorem 3 can be true in $P_{8}$-free graphs. Furthermore, observe that in the statements for $P_{5}$-free and $P_{6}$-free graphs the dominating set for the separator is guaranteed to be contained in two full components of the separator. This is no longer the case in Theorem 3 for a reason: in Section 5 we show examples of $P_{7}$-free graphs where any constant-size dominating set of a minimal separator needs to contain a vertex from the said separator.

The intuition behind the framework of PMCs, particularly visible in the quasi-polynomial-time algorithm for MWIS in $P_{6}$-free graphs [16], is that potential maximal cliques can serve as balanced separators of a graph. Here, $X \subseteq V(G)$ is a balanced separator of $G$ if every connected component of $G-X$ has at most $|V(G)| / 2$ vertices. The quasipolynomial-time algorithm of [16] tried to recursively split the graph into significantly smaller pieces by branching and deleting as large as possible pieces of such a PMC. Motivated by this intuition, in Section 4 we generalize Theorem 3 to dominating potential maximal cliques:

Theorem 4. Let $G$ be a $P_{7}$-free graph and let $\Omega$ be a potential maximal clique in $G$. Then there exists a set $\Omega^{\prime} \subseteq V(G)$ of size at most 68 such that $\Omega \subseteq N_{G}\left[\Omega^{\prime}\right]$.

Since every minimal separator is a subset of some potential maximal clique in a graph, Theorem 4 generalizes Theorem 3. For the same reason, our examples for $P_{8}$-free graphs also prohibit extending Theorem 4 to $P_{8}$-free graphs.

\section{Preliminaries}

For basic graph notation, we follow [13]. We outline here only nonstandard notation that is not presented in the introduction. 
For a set $X \subseteq V(G)$, by $\operatorname{cc}(G-X)$ we denote the family of connected components of $G-X$. A set $A$ is complete to a set $B$ if every vertex of $A$ is adjacent to every vertex of $B$.

Potential maximal cliques. A set $\Omega \subseteq V(G)$ is a potential maximal clique (PMC) if:

(PMC1) none of the connected components of $\operatorname{cc}(G-\Omega)$ is full to $\Omega$; and

(PMC2) whenever $u v$ is a non-edge with $\{u, v\} \subseteq \Omega$, then there is a component $D \in$ $\operatorname{cc}(G-\Omega)$ such that $\{u, v\} \subseteq N(D)$.

In the second condition, we will say that the component $D$ covers the non-edge $u v$. As announced in the introduction, we have the following.

Proposition 5 (Theorem 3.15 of [4]). For a graph $G$, a vertex subset $\Omega \subseteq V(G)$ is a $P M C$ if and only if there exists a minimal chordal completion $F$ of $G$ such that $\Omega$ is a maximal clique in $G+F$.

We will also need the following statement.

Lemma 6 (cf. Proposition 2.7 of [13], Proposition 8 of the arXiv version). For every $P M C \Omega$ of $G$ and every $D \in \mathrm{cc}(G-\Omega)$, the set $N_{G}(D)$ is a minimal separator.

Modules. Let $G$ be a graph. A set $M \subseteq V(G)$ is a module of $G$ if $N(x) \backslash M=N(y) \backslash M$ for every $x, y \in M$. Note that $\varnothing, V(G)$ and all the singletons $\{x\}$ for $x \in V(G)$ are modules; we call these modules trivial. A graph is prime if all its modules are trivial. A module $M$ of $G$ is strong if $M \neq V(G)$ and $M$ does not overlap with any other module of $G$, i.e., for every module $M^{\prime}$ of $G$ we have either $M \subseteq M^{\prime}$, or $M^{\prime} \subseteq M$, or $M \cap M^{\prime}=\varnothing$.

A partition $\mathcal{M}$ of $V(G)$ is a modular partition of $G$ if $M$ is a module of $G$ for every $M \in \mathcal{M}$. The quotient graph $G / \mathcal{M}$ is a graph with the vertex set $\mathcal{M}$ and with $M^{\prime} M \in$ $E(G / \mathcal{M})$ if and only if $m^{\prime} m \in E(G)$ for all $m^{\prime} \in M^{\prime}$ and $m \in M$ (since $M^{\prime}$ and $M$ are modules, $m^{\prime} m$ is an edge either for all pairs $m^{\prime} \in M^{\prime}$ and $m \in M$, or for none).

It is well-known (cf. [14, Lemma 2]) that if $|V(G)|>1$ then the family of (inclusionwise) maximal strong modules of $G$ forms a modular partition of $G$ whose quotient graph is either an independent set (if $G$ is not connected), a clique (if the complement of $G$ is not connected), or a prime graph (otherwise). We denote this modular partition by $\operatorname{Mod}(G)$ and we let $\operatorname{Quo}(G):=G / \operatorname{Mod}(G)$. For $D \subseteq V(G)$, we abbreviate $\operatorname{Mod}(G[D])$ and Quo $(G[D])$ with $\operatorname{Mod}(D)$ and $\mathrm{Quo}(D)$, respectively.

\section{Covering minimal separators in $\boldsymbol{P}_{7}$-free graphs}

This section is devoted to the proof of Theorem 3. We need the following two results from [13]. 
Lemma 7 (Bi-ranking Lemma, Lemma 4.1 of [13], Lemma 17 of the arXiv version). Suppose $X$ is a non-empty finite set and $\left(X, \leqslant_{1}\right)$ and $\left(X, \leqslant_{2}\right)$ are two quasi-orders. Suppose further that every pair of two different elements of $X$ is comparable either with respect to $\leqslant_{1}$ or with respect to $\leqslant_{2}$. Then there exists an element $x \in X$ such that for every $y \in X$ we have either $x \leqslant_{1} y$ or $x \leqslant_{2} y$.

Lemma 8 (Neighborhood Decomposition Lemma, Lemma 4.2 of [13], Lemma 18 of the arXiv version). Suppose $G$ is a graph and $D \subseteq V(G)$ is subset of vertices such that $|D| \geqslant 2$ and $G[D]$ is connected. Suppose further that vertices $p, q \in D$ respectively belong to different elements $M^{p}, M^{q}$ of the modular partition $\operatorname{Mod}(D)$ such that $M^{p}$ and $M^{q}$ are adjacent in the quotient graph Quo $(D)$. Then, for each vertex $u \in N(D)$ at least one of the following conditions holds:

(a) $u \in N[p, q]$;

(b) there exists an induced $P_{4}$ in $G$ such that $u$ is one of its endpoints, while the other three vertices belong to $D$;

(c) $\mathrm{Quo}(D)$ is a clique and the neighborhood of $u$ in $D$ is the union of some collection of maximal strong modules in $D$.

In particular, if $\mathrm{Quo}(D)$ is not a clique, then the last condition cannot hold.

Let $G$ be a $P_{7}$-free graph, let $S$ be a minimal separator in $G$, and let $A_{1}$ and $A_{2}$ be the vertex sets of two full components of $S$. If $\left|A_{i}\right|=1$ for some $i \in\{1,2\}$, then Theorem 3 holds by setting $S^{\prime}:=A_{i}$, so we may assume $\left|A_{1}\right|,\left|A_{2}\right|>1$. For each $i \in\{1,2\}$, fix two different maximal strong modules $M_{i}^{p}$ and $M_{i}^{q}$ of $G\left[A_{i}\right]$ that are adjacent in Quo $\left(A_{i}\right)$. Furthermore, pick arbitrary $p_{i} \in M_{i}^{p}$ and $q_{i} \in M_{i}^{q}$.

For each $i \in\{1,2\}$, we apply Lemma 8 to $D:=A_{i}$ and $N(D)=S$. We say that a vertex $x \in S$ is of type $(a)_{i}$ if $x \in N\left(p_{i}\right) \cup N\left(q_{i}\right)$. We say that a vertex $x \in S$ is of type $(b)_{i}$ if $x$ is not of type $(a)_{i}$ and there is an induced $P_{4}$ in $G$ with $x$ being one of the endpoints and the other three vertices belonging to $A_{i}$. Finally, we say that a vertex $x \in S$ is of type $(c)_{i}$ if $x$ is neither of type $(a)_{i}$ nor $(b)_{i}$. Lemma 8 asserts that if there are vertices of type $(c)_{i}$, then Quo $\left(A_{i}\right)$ is a clique and the neighborhood in $A_{i}$ of every vertex of this type is the union of a collection of maximal strong modules of $G\left[A_{i}\right]$. For $\alpha, \beta \in\{a, b, c\}$, let $S_{\alpha \beta}$ be the set of vertices $x \in S$ that are of type $(\alpha)_{1}$ and $(\beta)_{2}$.

We need the following claim.

Claim 9. Let $i \in\{1,2\}$ and let $x, y \in S$ be of type $(c)_{i}$. Then $A_{i} \cap(N(x) \backslash N(y))$ is complete to $A_{i} \cap(N(y) \backslash N(x))$.

Proof. By Lemma 8, Quo $\left(A_{i}\right)$ is a clique and both $A_{i} \cap(N(x) \backslash N(y))$ and $A_{i} \cap(N(y) \backslash N(x))$ are the unions of some disjoint collections of maximal strong modules of $G\left[A_{i}\right]$. The claim follows. 
Since $G$ is $P_{7}$-free, $S_{b b}=\varnothing$. Furthermore, if we set $R_{a}:=\left\{p_{1}, q_{1}, p_{2}, q_{2}\right\}$, then

$$
S_{a a} \cup S_{a b} \cup S_{a c} \cup S_{b a} \cup S_{c a} \subseteq N\left(R_{a}\right) .
$$

In the rest of the proof, we construct sets $R_{b c}, R_{c b}$ and $R_{c c}$ such that $S_{\alpha \beta} \subseteq N\left[R_{\alpha \beta}\right]$ for $\alpha \beta \in\{b c, c b, c c\}$. We will conclude that $S^{\prime}:=R_{a} \cup R_{b c} \cup R_{c b} \cup R_{c c}$ satisfies the statement of the lemma, because $\left|R_{a}\right|=4$ and we will ensure that $\left|R_{b c}\right|,\left|R_{c b}\right| \leqslant 5$ and $\left|R_{c c}\right| \leqslant 8$.

We start with constructing the set $R_{b c}$. If $S_{b c}=\varnothing$, then we set $R_{b c}=\varnothing$. Otherwise, let $v \in S_{b c}$ be a vertex with inclusion-wise minimal set $A_{2} \cap N(v)$. Furthermore, let $w \in A_{2}$ be an arbitrary neighbor of $v$ in $A_{2} ; w$ exists since $A_{2}$ is a full component of $S$. Also, let $v, u^{1}, u^{2}, u^{3}$ be vertices of an induced $P_{4}$ with $u^{1}, u^{2}, u^{3} \in A_{1}$; recall here that $v$ is of type $(b)_{1}$. We set $R_{b c}:=\left\{u^{1}, u^{2}, u^{3}, v, w\right\}$ and claim that $S_{b c} \subseteq N\left[R_{b c}\right]$.

Assume the contrary, and let $v^{\prime} \in S_{b c} \backslash N\left[R_{b c}\right]$. By the choice of $v$ and since $w \in$ $A_{2} \cap\left(N(v) \backslash N\left(v^{\prime}\right)\right)$, there exists $w^{\prime} \in A_{2} \cap\left(N\left(v^{\prime}\right) \backslash N(v)\right)$. By Claim 9, ww $\in E(G)$. Then, $v^{\prime}-w^{\prime}-w-v-u^{1}-u^{2}-u^{3}$ is an induced $P_{7}$ in $G$, a contradiction.

Hence, we constructed $R_{b c} \subseteq V(G)$ of size at most 5 such that $S_{b c} \subseteq N\left[R_{b c}\right]$. A symmetric reasoning yields $R_{c b} \subseteq V(G)$ of size at most 5 such that $S_{c b} \subseteq N\left[R_{c b}\right]$.

We are left with constructing $R_{c c}$. If $S_{c c}=\varnothing$, then we take $R_{c c}=\varnothing$ and conclude. In the remaining case, $S_{c c}$ is non-empty, so both Quo $\left(A_{1}\right)$ and Quo $\left(A_{2}\right)$ are cliques.

For each $i \in\{1,2\}$, we define a quasi-order $\leqslant_{i}$ on $S_{c c}$ as follows. For $x, y \in S_{c c}, x \leqslant_{i} y$ if $A_{i} \cap N(x) \subseteq A_{i} \cap N(y)$. An unordered pair $x y \in\left(\begin{array}{c}S_{c c} \\ 2\end{array}\right)$ is a butterfly if $x$ and $y$ are incomparable both in $\leqslant_{1}$ and in $\leqslant_{2}$, that is, if each of the following four sets is nonempty:

$$
\begin{array}{ll}
A_{1} \cap(N(x) \backslash N(y)), & A_{1} \cap(N(y) \backslash N(x)), \\
A_{2} \cap(N(x) \backslash N(y)), & A_{2} \cap(N(y) \backslash N(x)) .
\end{array}
$$

See Figure 1 for an illustration.

Lemma 7 allows us to easily dominate subsets of $S_{c c}$ that do not contain any butterflies:

Claim 10. Let $T \subseteq S_{c c}$ be such that there is no butterfly $x y$ with $x, y \in T$. Then there exist vertices $a_{1} \in A_{1}$ and $a_{2} \in A_{2}$ such that $T \subseteq N\left(a_{1}\right) \cup N\left(a_{2}\right)$.

Proof. If $T=\varnothing$, the claim is trivial, so assume otherwise. Let us focus on quasi-orders $\leqslant 1$ and $\leqslant_{2}$, restricted to $T$. Since there are no butterflies in $T$, the prerequisities of Lemma 7 are satisfied for $\left(T, \leqslant_{1}\right)$ and $\left(T, \leqslant_{2}\right)$. Hence, there exists $x \in T$ with $x \leqslant_{1} y$ or $x \leqslant_{2} y$ for every $y \in T$. For $i \in\{1,2\}$, let $a_{i}$ be an arbitrary neighbor of $x$ in $A_{i}$ (it exists as $A_{i}$ is a full component of $S$ ). For every $y \in T$, there exists $i \in\{1,2\}$ such that $x \leqslant_{i} y$, hence $a_{i} y \in E(G)$. We conclude that $T \subseteq N\left(a_{1}\right) \cup N\left(a_{2}\right)$, as desired.

If there is no butterfly at all, then we apply Claim 10 to $T=S_{c c}$, obtaining vertices $a_{1}, a_{2}$ and set $R_{c c}=\left\{a_{1}, a_{2}\right\}$. Thus, we are left with the case where at least one butterfly exists.

Let $x y$ be a butterfly with inclusion-wise minimal set

$$
\left(A_{1} \cup A_{2}\right) \cap N(\{x, y\}) .
$$




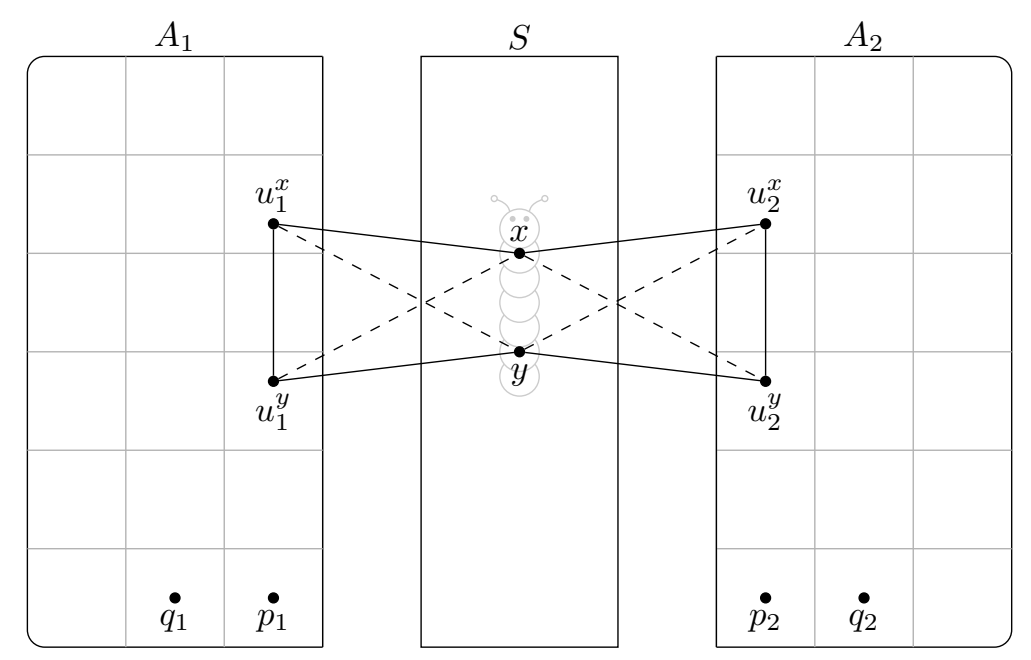

Figure 1: A butterfly $x y$. The gray grid presents the partion of $G\left[A_{i}\right]$ into maximal strong modules.

Furthermore, pick the following four vertices

$$
\begin{array}{ll}
u_{1}^{x} \in A_{1} \cap(N(x) \backslash N(y)), & u_{1}^{y} \in A_{1} \cap(N(y) \backslash N(x)), \\
u_{2}^{x} \in A_{2} \cap(N(x) \backslash N(y)), & u_{2}^{y} \in A_{2} \cap(N(y) \backslash N(x)) .
\end{array}
$$

Claim 9 ensures that $u_{1}^{x} u_{1}^{y} \in E(G)$ and $u_{2}^{x} u_{2}^{y} \in E(G)$.

Set

$$
R^{\prime}:=\left\{x, y, u_{1}^{x}, u_{1}^{y}, u_{2}^{x}, u_{2}^{y}\right\}
$$

Let $T:=S_{c c} \backslash N\left[R^{\prime}\right]$. We claim the following:

Claim 11. There is no butterfly $x^{\prime} y^{\prime}$ with $x^{\prime}, y^{\prime} \in T$.

Proof. Assume the contrary, and let $x^{\prime} y^{\prime}$ be a butterfly with $x^{\prime}, y^{\prime} \in T$. By the minimality of $x y$, as $u_{1}^{x} \in\left(A_{1} \cup A_{2}\right) \cap N(\{x, y\})$ but $u_{1}^{x} \notin\left(A_{1} \cup A_{2}\right) \cap N\left(\left\{x^{\prime}, y^{\prime}\right\}\right)$, there exists $w \in\left(A_{1} \cup A_{2}\right) \cap\left(N\left(\left\{x^{\prime}, y^{\prime}\right\}\right) \backslash N(\{x, y\})\right)$. By symmetry, assume that $w \in A_{1}$ and $x^{\prime} w \in E(G)$; see Figure 2 .

By Claim 9, $w u_{1}^{x} \in E(G)$ and $w u_{1}^{y} \in E(G)$. If $x y \in E(G)$, then $x^{\prime}-w-u_{1}^{x}-x-y-u_{2}^{y}-$ $p_{2}$ would be an induced $P_{7}$ in $G$. Otherwise, if $x y \notin E(G)$, then $x^{\prime}-w-u_{1}^{x}-x-u_{2}^{x}-u_{2}^{y}-y$ would be an induced $P_{7}$ in $G$. As in both cases we have obtained a contradiction, this finishes the proof.

By Claim 11, we can apply Claim 10 to $T$ and obtain vertices $a_{1} \in A_{1}$ and $a_{2} \in A_{2}$ with $T \subseteq N\left(a_{1}\right) \cup N\left(a_{2}\right)$. Hence, we can take $R_{c c}:=R^{\prime} \cup\left\{a_{1}, a_{2}\right\}$, thus concluding the proof of Theorem 3 . 

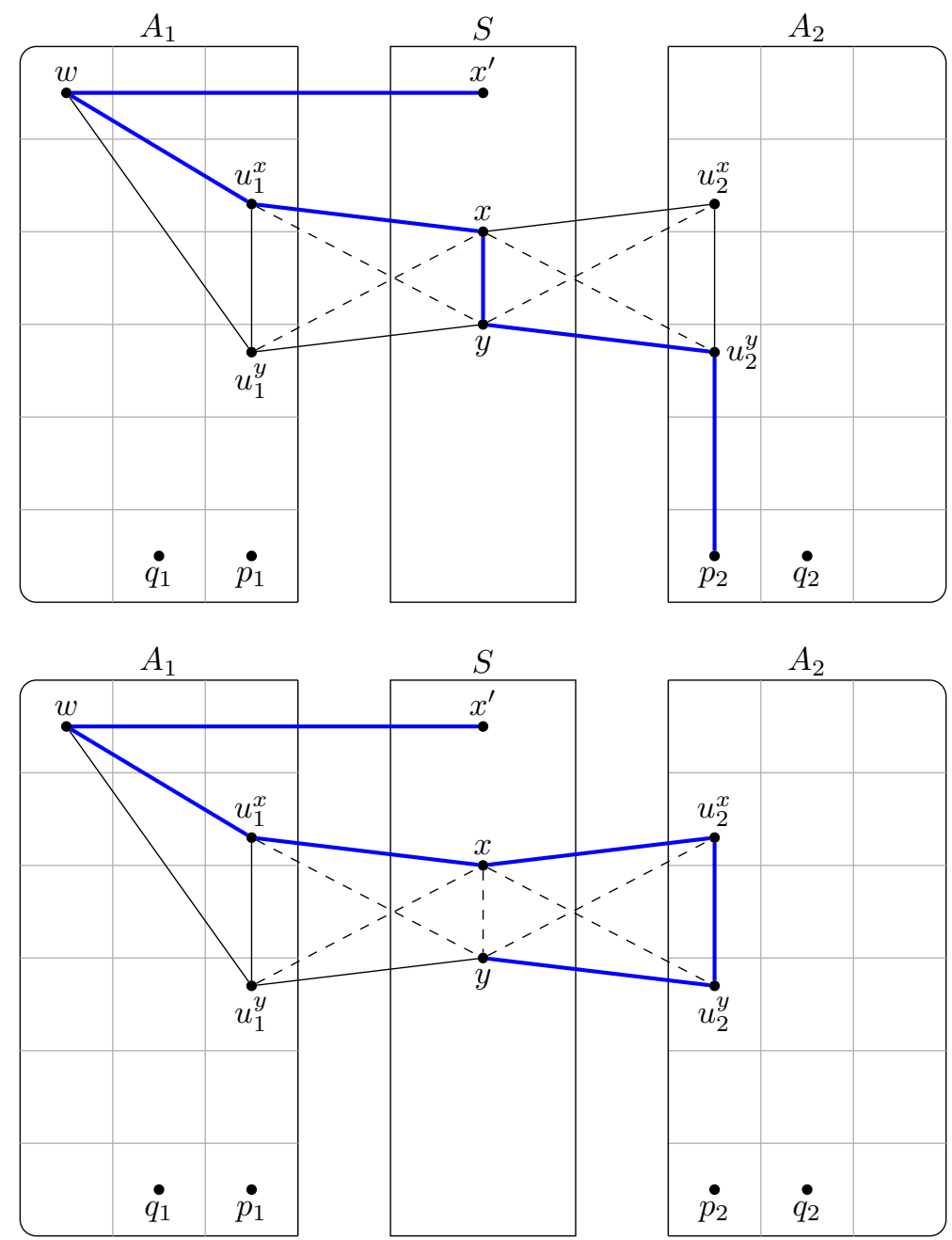

Figure 2: Two cases where a $P_{7}$ appears in the proof of Claim 11.

\section{Covering PMCs in $P_{7}$-free graphs}

We now prove the following statement which, together with Theorem 3 and Lemma 6 , immediately implies Theorem 4.

Lemma 12. Let $G$ be a $P_{7}$-free graph and let $\Omega$ be a potential maximal clique in $G$. Then there exists a set $\Omega^{\prime} \subseteq \Omega$ of size at most 2 and a set $\mathcal{D}^{\prime} \subseteq \operatorname{cc}(G-\Omega)$ of size at most 3 such that

$$
\Omega \subseteq N\left[\Omega^{\prime}\right] \cup \bigcup_{D \in \mathcal{D}^{\prime}} N(D)
$$

Proof. Let $\mathcal{D} \subseteq \operatorname{cc}(G-\Omega)$ be an inclusion-wise minimal set of components of $G-\Omega$ such that for every nonedge $u v$ in $\Omega$ there exists a component $D \in \mathcal{D}$ that covers $u v$.

If $\mathcal{D}=\varnothing$, then $\Omega$ is a clique in $G$ and thus we can put $\Omega^{\prime}=\{v\}$ and $\mathcal{D}^{\prime}=\varnothing$ for an 
arbitrary $v \in \Omega$. Otherwise, pick any $D \in \mathcal{D}$. By the minimality of $\mathcal{D}$, there exists a nonedge $u v$ in $\Omega$ that is covered by $D$ and by no other component of $\mathcal{D} \backslash\{D\}$.

Assume that there is no component $D_{v} \in \mathcal{D} \backslash\{D\}$ with $v \in N\left(D_{v}\right)$. Then $\Omega \backslash N(D) \subseteq$ $N(v)$, so $\Omega \subseteq N(v) \cup N(D)$. Hence, we can set $\Omega^{\prime}=\{v\}$ and $\mathcal{D}^{\prime}=\{D\}$. Symmetrically, Lemma 12 holds if there is no component $D_{u} \in \mathcal{D} \backslash\{D\}$ with $u \in N\left(D_{u}\right)$.

In the remaining case, pick arbitrary components $D_{v} \in \mathcal{D} \backslash\{D\}$ with $v \in N\left(D_{v}\right)$ and $D_{u} \in \mathcal{D} \backslash\{D\}$ with $u \in N\left(D_{u}\right)$. Since $D$ is the only component of $\mathcal{D}$ that covers $u v$, we have $u \notin N\left(D_{v}\right)$ and $v \notin N\left(D_{u}\right)$; in particular, $D_{u} \neq D_{v}$. We claim that we can set $\Omega^{\prime}=\{u, v\}$ and $\mathcal{D}^{\prime}=\left\{D, D_{u}, D_{v}\right\}$. That is, we claim that

$$
\Omega \subseteq N[u] \cup N[v] \cup N(D) \cup N\left(D_{u}\right) \cup N\left(D_{v}\right) .
$$

Assume the contrary, and let $x \in \Omega$ be such that $x u \notin E(G), x v \notin E(G), x \notin N(D)$, $x \notin N\left(D_{u}\right)$, and $x \notin N\left(D_{v}\right)$.

Since $x u$ is a nonedge of $\Omega$, there exists $D_{x u} \in \mathcal{D}$ that covers $x u$. Similarly, there exists $D_{x v} \in \mathcal{D}$ that covers $x v$. By the choice of $x$, we have $D_{x u}, D_{x v} \notin\left\{D, D_{u}, D_{v}\right\}$. Further, since $D$ is the only component of $\mathcal{D}$ covering $u v, v \notin N\left(D_{x u}\right)$ and $u \notin N\left(D_{x v}\right)$; in particular, $D_{x u} \neq D_{x v}$. See Figure 3 .

Let $y_{u}$ be an arbitrary neighbor of $u$ in $D_{u}$, let $y_{v}$ be an arbitrary neighbor of $v$ in $D_{v}$, let $P_{u}$ be a shortest path from $u$ to $x$ with all internal vertices in $D_{x u}$, and let $P_{v}$ be a shortest path from $v$ to $x$ with all internal vertices in $D_{x v}$. Then, $y_{u}-u-P_{u}-x-P_{v}-v-y_{v}$ is an induced path with at least 7 vertices, a contradiction. This proves (1) and concludes the proof of Lemma 12.

\section{$5 \quad$ Examples}

In this section we discuss two examples showing tightness of the statement of Theorem 3 : we show that it cannot be generalized to $P_{8}$-free graphs and that a small dominating set of a minimal separator may need to contain elements of the said separator. The examples are modifications of a corresponding example presented in the conclusions of [16].

First example. Consider the following graph $G$. We create three sets of $n$ vertices each, $A_{1}=\left\{a_{1}^{j} \mid 1 \leqslant j \leqslant n\right\}, A_{2}=\left\{a_{2}^{j} \mid 1 \leqslant j \leqslant n\right\}$, and $S=\left\{s^{j} \mid 1 \leqslant j \leqslant n\right\}$. We set $V(G)=A_{1} \cup A_{2} \cup S$. For the edge set of $G$, we turn $A_{1}$ and $A_{2}$ into cliques and add edges $s^{j} a_{1}^{j}$ and $s^{j} a_{2}^{j}$, for all $1 \leqslant j \leqslant n$. This concludes the description of the graph $G$; see Figure 4. Note that $S$ is a minimal separator in $G$ with $A_{1}$ and $A_{2}$ being two full components of $S$.

First, note that for every $v \in V(G),|N[v] \cap S|=1$. Thus, any set dominating $S$ has to contain at least $n$ vertices.

Second, note that $G$ is $P_{8}$-free. To see this, let $P$ be an induced path in $G$. Since $A_{1}$ and $A_{2}$ are cliques, $P$ contains at most two vertices from each $A_{i}, i \in\{1,2\}$, and these vertices are consecutive on $P$. Since $S$ is an independent set in $G, P$ cannot contain more than one vertex of $S$ in a row. Hence, $P$ contains at most three vertices of $S$. 


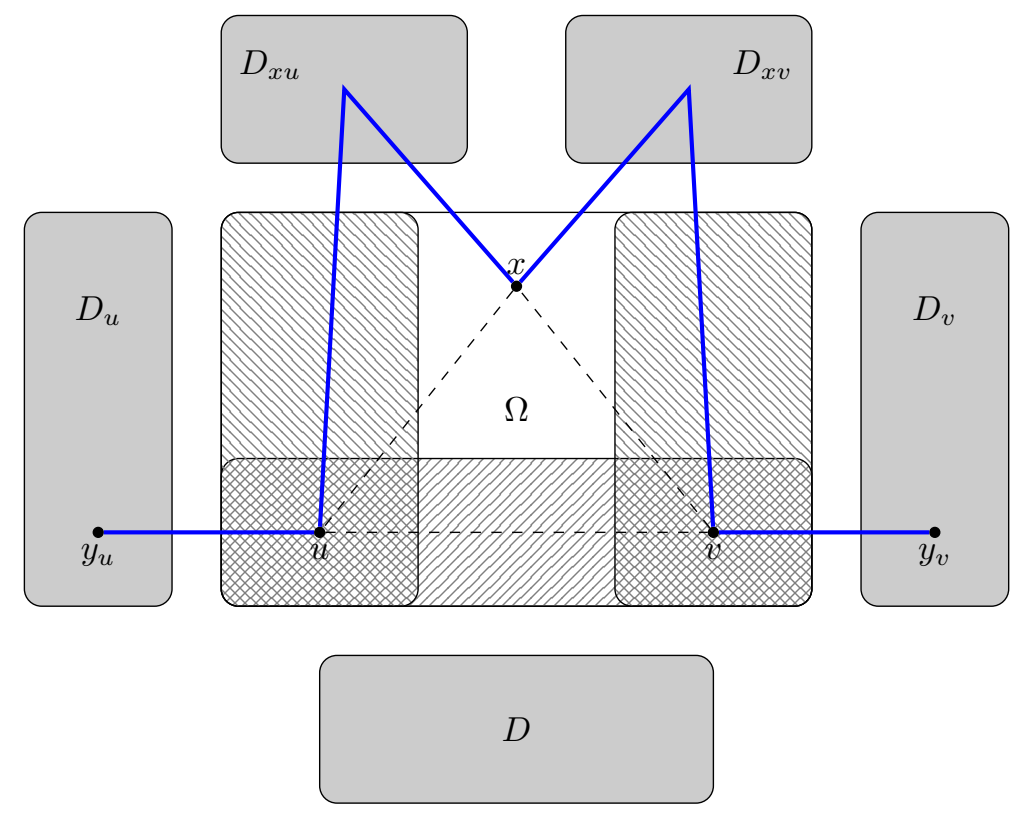

Figure 3: A $P_{7}$ in the proof of Lemma 12. $N(D)$ is the horizontal box inside $\Omega$ marked with north-east strips and $N\left(D_{u}\right) \cup N\left(D_{v}\right)$ consist of two vertical boxes inside $\Omega$ marked with north-west strips. Note that $N\left(D_{u}\right) \cap N\left(D_{v}\right)$ may be nonempty, which is not depicted on the figure.

Consequently $|V(P)| \leqslant 7$, as desired. Note that if $n \geqslant 3$, then there is an induced $P_{7}$ in $G$, for example $s^{1}-a_{1}^{1}-a_{1}^{2}-s^{2}-a_{2}^{2}-a_{2}^{3}-s^{3}$.

Second example. Here, let us modify the graph $G$ from the first example by turning $S$ into a clique. Still, $S$ is a minimal separator in $G$ with $A_{1}$ and $A_{2}$ being two full components of $S$.

First, note that for every $v \in A_{1} \cup A_{2}$, we still have $|N[v] \cap S|=1$. Thus, any set dominating $S$ that is disjoint with $S$ has to contain at least $n$ vertices.

Second, note that $G$ is $P_{7}$-free. To see this, observe that $G$ can be partitioned into three cliques, $A_{1}, A_{2}$, and $S$, and any induced path in $G$ contains at most two vertices from each of the cliques. Note that if $n \geqslant 3$, then there is an induced $P_{6}$ in $G$, for example $a_{1}^{3}-a_{1}^{1}-s^{1}-s^{2}-a_{2}^{2}-a_{2}^{3}$.

While the two examples above refute the possibility of covering a minimal separator or a PMC by a constant number of vertex neighborhoods, they do not refute a weaker statement such as the one of Lemma 12: covering a PMC with a constant number of vertex or component neighborhoods. We were not able to construct a counterexample to such a statement. On the contrary, we conjecture the following:

Conjecture 13. For every integer $t \geqslant 2$ there exists an integer $M_{t}$ such that for every $P_{t^{-}}$ free graph $G$ and every PMC $\Omega$ in $G$, there exists a set $X \subseteq V(G)$ and a set $\mathcal{D} \subseteq \operatorname{cc}(G-\Omega)$, both of size at most $M_{t}$, such that $\Omega \subseteq N[X] \cup \bigcup_{D \in \mathcal{D}} N(D)$. 


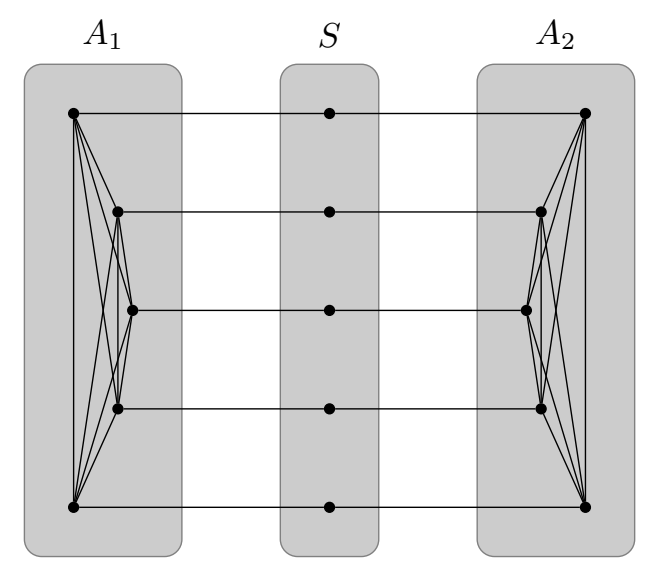

Figure 4: The first example for $n=5$. In the second example we turn $S$ into a clique.

Lemma 12 provides the proof for $t=7$ with $M_{7}=3$.

In support of the above conjecture let us recall the following technical statement of [16]:

Lemma 14 (rephrasing of Lemma 3.3 of [16]). Let $H$ be a graph on $n_{H}$ vertices and $m_{H}$ edges. Then, for every graph $G$, every $P M C \Omega$ in $G$, and every probabilistic distribution $\mu$ on $\Omega$, there exists at least one of the following:

1. a vertex $v \in V(G)$ with $\mu(N[v] \cap \Omega) \geqslant \frac{1}{2 n_{H}^{2}}$;

2. a component $D \in \mathrm{cc}(G-\Omega)$ with $\mu(N(D)) \geqslant \frac{1}{2 n_{H} m_{H}}$; or

3. an induced subgraph of $G$ isomorphic to a subdivision of $H$.

Observe that an iterative application of Lemma 14 with $H=P_{t}$ to a $P_{t}$-free graph $G$ and a PMC $\Omega$ yields a weaker version of Conjecture 13:

Lemma 15. For every $t$, every $P_{t}$-free graph $G$, and every $P M C \Omega$ in $G$, there exists a set $X \subseteq V(G)$ and a set $\mathcal{D} \subseteq \operatorname{cc}(G-\Omega)$, both of size $\mathcal{O}\left(t^{2} \log |\Omega|\right)$, such that $\Omega \subseteq$ $N[X] \cup \bigcup_{D \in \mathcal{D}} N(D)$.

Proof. We compute $X$ and $\mathcal{D}$ via the following process, initiated with $X=\varnothing$ and $\mathcal{D}=\varnothing$. At each step, given $X \subseteq V(G)$ and $\mathcal{D} \subseteq \mathrm{cc}(G-\Omega)$, compute $A:=\Omega \backslash\left(N[X] \cup \bigcup_{D \in \mathcal{D}} N(D)\right)$. If $A=\varnothing$, then stop, returning $X$ and $\mathcal{D}$. Otherwise, apply Lemma 14 to $H=P_{t}, G, \Omega$, and $\mu$ being a uniform distribution over $A$. Since $G$ is $P_{t^{-}}$free, Lemma 14 finds a vertex $v$ with $\mu(N[v] \cap \Omega) \geqslant 1 / 2 t^{2}$ or a component $D \in \operatorname{cc}(G-\Omega)$ with $\mu(N(D)) \geqslant 1 / 2 t^{2}$. In the first case, add $v$ to $X$, and in the second case, add $D$ to $\mathcal{D}$, and go to the next step. Observe that by the choice of $\mu$, in the next iteration of the process the set $A$ is smaller by at least a factor of $\left(1-1 / 2 t^{2}\right)$, proving the final bound on $|X|$ and $|\mathcal{D}|$.

However, we remark that it is unclear whether a positive resolution of Conjecture 13 would have any application for polynomial-time algorithms in the class of $P_{t}$-free graphs. 
Acknowledgements. We thank the reviewers for multiple comments that helped in improving this article, in particular for raising a question that led to the statement of Conjecture 13.

\section{References}

[1] V. E. Alekseev. The effect of local constraints on the complexity of determination of the graph independence number. Combinatorial-algebraic methods in applied mathematics, pages 3-13, 1982. (in Russian).

[2] G. Bacsó, D. Lokshtanov, D. Marx, M. Pilipczuk, Z. Tuza, and E. J. van Leeuwen. Subexponential-time algorithms for Maximum Independent Set in $P_{t}$-free and broomfree graphs. Algorithmica, 81(2):421-438, 2019.

[3] F. Bonomo, M. Chudnovsky, P. Maceli, O. Schaudt, M. Stein, and M. Zhong. Threecoloring and list three-coloring of graphs without induced paths on seven vertices. Combinatorica, 38(4):779-801, 2018.

[4] V. Bouchitté and I. Todinca. Treewidth and Minimum Fill-in: Grouping the minimal separators. SIAM J. Comput., 31(1):212-232, 2001.

[5] V. Bouchitté and I. Todinca. Listing all potential maximal cliques of a graph. Theor. Comput. Sci., 276(1-2):17-32, 2002.

[6] C. Brause. A subexponential-time algorithm for the Maximum Independent Set problem in $P_{t}$-free graphs. Discret. Appl. Math., 231:113-118, 2017.

[7] M. Chudnovsky, M. Pilipczuk, M. Pilipczuk, and S. Thomassé. Quasi-polynomial time approximation schemes for the Maximum Weight Independent Set problem in $H$-free graphs. In Proceedings of the $31^{\text {st }}$ ACM-SIAM Symposium on Discrete Algorithms, SODA 2020, pages 2260-2278. SIAM, 2020.

[8] M. Chudnovsky, S. Spirkl, and M. Zhong. Four-coloring $P_{6}$-free graphs. In Proceedings of the $30^{\text {th }}$ Annual ACM-SIAM Symposium on Discrete Algorithms, SODA 2019, pages 1239-1256. SIAM, 2019.

[9] F. V. Fomin, I. Todinca, and Y. Villanger. Large induced subgraphs via triangulations and CMSO. SIAM J. Comput., 44(1):54-87, 2015.

[10] P. Gartland and D. Lokshtanov. Independent Set on $P_{k}$-free graphs in quasipolynomial time. In IEEE $61^{\text {st }}$ Annual Symposium on Foundations of Computer Science, FOCS 2020, pages 612-624, 2020.

[11] P. A. Golovach, D. Paulusma, and J. Song. Closing complexity gaps for coloring problems on h-free graphs. Inf. Comput., 237:204-214, 2014.

[12] C. Groenland, K. Okrasa, P. Rzążewski, A. D. Scott, P. D. Seymour, and S. Spirkl. $H$-colouring $P_{t^{-}}$-free graphs in subexponential time. Discret. Appl. Math., 267:184189, 2019. 
[13] A. Grzesik, T. Klimošová, M. Pilipczuk, and M. Pilipczuk. Polynomial-time algorithm for Maximum Weight Independent Set on $P_{6}$-free graphs. ACM Trans. Algorithms, 2020. In press arXiv:1707.05491.

[14] M. Habib and C. Paul. A survey of the algorithmic aspects of modular decomposition. Computer Science Review, 4(1):41-59, 2010.

[15] C. T. Hoàng, M. Kamiński, V. V. Lozin, J. Sawada, and X. Shu. Deciding kcolorability of $P_{5}$-free graphs in polynomial time. Algorithmica, 57(1):74-81, 2010.

[16] D. Lokshtanov, M. Pilipczuk, and E. J. van Leeuwen. Independence and efficient domination on $P_{6}$-free graphs. ACM Trans. Algorithms, 14(1):3:1-3:30, 2018.

[17] D. Lokshtanov, M. Vatshelle, and Y. Villanger. Independent set in $P_{5}$-free graphs in polynomial time. In Proceedings of the $25^{\text {th }}$ Annual ACM-SIAM Symposium on Discrete Algorithms, SODA 2014, pages 570-581. SIAM, 2014.

[18] V. V. Lozin and M. Milanič. A polynomial algorithm to find an independent set of maximum weight in a fork-free graph. J. Discrete Algorithms, 6(4):595-604, 2008.

[19] G. J. Minty. On maximal independent sets of vertices in claw-free graphs. J. Comb. Theory, Ser. B, 28(3):284-304, 1980.

[20] M. Pilipczuk, M. Pilipczuk, and P. Rzążewski. Quasi-polynomial-time algorithm for Independent Set in $P_{t}$-free graphs via shrinking the space of induced paths. In Proceedings of the SIAM Symposium on Symplicity in Algorithms, SOSA 2021, pages 204-209, 2021.

[21] N. Sbihi. Algorithme de recherche d'un stable de cardinalite maximum dans un graphe sans etoile. Discrete Mathematics, 29(1):53-76, 1980. (in French). 